\title{
AN ERROR BOUND ANALYSIS FOR CUBIC SPLINE APPROXIMATION OF CONIC SECTION
}

\author{
YOUNG JOON AHN
}

\begin{abstract}
In this paper we present an error bound for cubic spline approximation of conic section curve. We compare it to the error bound proposed by Floater [1]. The error estimating function proposed in this paper is sharper than Floater's at the mid-point of parameter, which means the overall error bound is sharper than Floater's if the estimating function has the maximum at the midpoint.
\end{abstract}

\section{Introduction}

The conversion problem of the rational splines into the (non-rational) splines is one of the most important requirements in CAD or CAGD. Many results for this topic have been published $[2,3,4,5,6]$, but none of them could obtain the closed error form of optimal convergence order except for special cases. In the spline approximation schemes for the special case of rational curves, e.g., circular arcs $[7,8,9,10,11]$ and conic sections $[1,12,13]$, the closed error forms of optimal order of convergence have been presented. Especially, Floater used the implicit form of conic section [14]

$$
\tau_{1}^{2}-4 \mu^{2} \tau_{0} \tau_{2}=0
$$

where $\mu=w_{1} / \sqrt{w_{0} w_{2}}$ is the fullness factor $[15,16,17], w_{0}, w_{1}, w_{2}$ are the weights of the rational Bézier representation of the curve and $\tau_{0}, \tau_{1}, \tau_{2}$ are the barycentric coordinates with respect to the control points $\mathbf{b}_{0}, \mathbf{b}_{1}, \mathbf{b}_{2}$ of the conic. In this paper, we use the implicit form

$$
\tau_{1}-2 \mu \sqrt{\tau_{0} \tau_{2}}=0,
$$

Received August 20, 2001.

2000 Mathematics Subject Classification: 41A50, 41A52, 65D05, 64D07.

Key words and phrases: conic section; spline approximation; cubic Beźier curve; error analysis; geometric Hermite interpolation. 
to obtain another error bound. In case the error function has its maximum at the mid-point of parameter, we can see that our error analysis give the error bounds sharper than Floater's analysis [1]. We apply our error analysis to the cubic spline approximations for conic sections and compare our error bounds to the former works [1], numerically. All error analysis discussed in this paper can be extended to tensor product surfaces, as well as to polynomial approximation of higher degree than three, if the error estimating function proposed in this paper has the maximum at the mid-point of parameter.

\section{Distance from conic section to the approximation curve}

In this section we consider the distance between the approximation curve and given the conic section represented in the standard rational quadratic Bézier form

$$
\mathbf{r}(t)=\frac{B_{0}^{2}(t) \mathbf{p}_{0}+B_{1}^{2}(t) \mu \mathbf{p}_{1}+B_{2}^{2}(t) \mathbf{p}_{2}}{B_{0}^{2}(t)+B_{1}^{2}(t) \mu+B_{2}^{2}(t)}, \quad t \in[0,1]
$$

where $\mathbf{p}_{0}, \mathbf{p}_{1}, \mathbf{p}_{2} \in \mathbf{R}^{2}$ are the control points, $\mu>0$ is the weight associated with $\mathbf{p}_{1}$, and $B_{i}^{n}(t)$ is the Bernstein polynomial of degree $n$ given by

$$
B_{i}^{n}(t)=\frac{n !}{i !(n-i) !} t^{i}(1-t)^{n-i}
$$

Any point $(x, y) \in \triangle \mathbf{p}_{0} \mathbf{p}_{1} \mathbf{p}_{2}$ can be written uniquely in terms of barycentric coordinates $\tau_{0}, \tau_{1}, \tau_{2}$, where $\tau_{0}+\tau_{1}+\tau_{2}=1$, with respect to $\triangle \mathbf{p}_{0} \mathbf{p}_{1} \mathbf{p}_{2}:(x, y)=\tau_{0} \mathbf{p}_{0}+\tau_{1} \mathbf{p}_{1}+\tau_{2} \mathbf{p}_{2}$. Consequently any function can be expressed as a function of $\tau_{0}, \tau_{1}, \tau_{2}$. We define the functions $f_{0}$ and $f_{1}: \triangle \mathbf{p}_{0} \mathbf{p}_{1} \mathbf{p}_{2} \rightarrow \mathbf{R}$ by

(1) $\quad f_{0}(x, y)=\tau_{1}^{2}-4 \mu^{2} \tau_{0} \tau_{2} \quad$ and $\quad f_{1}(x, y)=\tau_{1}-2 \mu \sqrt{\tau_{0} \tau_{2}}$.

Since $0 \leq \tau_{i} \leq 1, i=0,1,2$, for each $(x, y) \in \triangle \mathbf{p}_{0} \mathbf{p}_{1} \mathbf{p}_{2}$, the functions $f_{0}$ and $f_{1}$ are well-defined in $\triangle \mathbf{p}_{0} \mathbf{p}_{1} \mathbf{p}_{2}$, especially, $f_{0}$ is defined in the whole $x y$-plane [14]. The following lemma is also a well-known fact [14].

Lemma 2.1. Let $f_{j}: \triangle \mathbf{p}_{0} \mathbf{p}_{1} \mathbf{p}_{2} \rightarrow \mathbf{R}$ be defined as in Equation (1). For $t \in[0,1]$, the point $\mathbf{r}(t)$ satisfies the equation $f_{j}(\mathbf{r}(t))=0, j=0,1$.

For any two plane curves $\mathbf{c}(s)$ and $\mathbf{q}(t), s, t \in[0,1]$, it is not easy to find the Hausdorff distance $d_{H}(\mathbf{c}, \mathbf{q})$ in general, where

$$
d_{H}(\mathbf{c}, \mathbf{q})=\max \left\{\max _{s} \min _{t}|\mathbf{c}(s)-\mathbf{q}(t)|, \max _{t} \min _{s}|\mathbf{c}(s)-\mathbf{q}(t)|\right\} .
$$




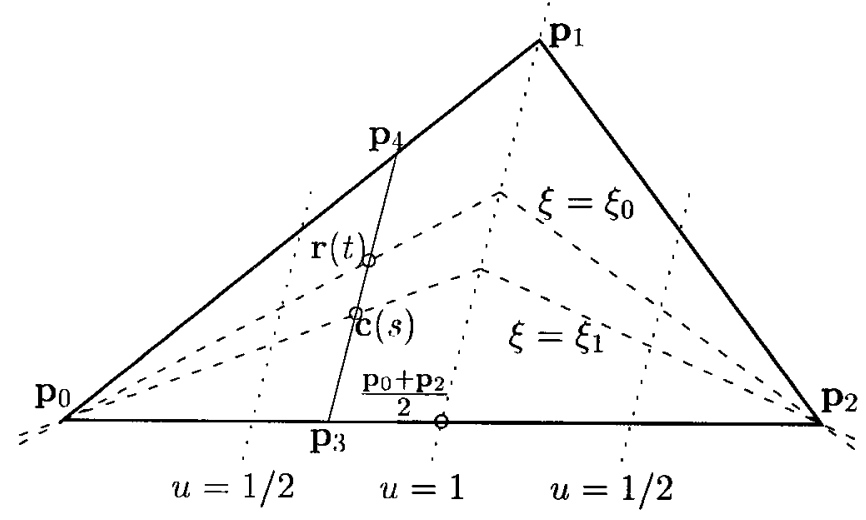

Figure 1 . In the triangle $\Delta \mathbf{p}_{0} \mathbf{p}_{1} \mathbf{p}_{2}$, the coordinates $(u, \xi)$ is symmetric with respect to the line passing through $\mathbf{p}_{1}$ and $\frac{\mathbf{p}_{0}+\mathbf{p}_{2}}{2}$.

For any onto function $\phi:[0,1] \rightarrow[0,1]$ it is clear that

$$
\max _{s} \min _{t}|\mathbf{c}(s)-\mathbf{q}(t)| \leq \max _{s}|\mathbf{c}(s)-\mathbf{q}(\phi(s))| .
$$

For each $t \in[0,1]$, let $s^{\prime} \in[0,1]$ be a value satisfying $\phi\left(s^{\prime}\right)=t$. (It is possible since $\phi$ is onto.) Then

(3) $\max _{t} \min _{s}|\mathbf{c}(s)-\mathbf{q}(t)| \leq \max _{t}\left|\mathbf{c}\left(s^{\prime}\right)-\mathbf{q}(t)\right|=\max _{s^{\prime}}\left|\mathbf{c}\left(s^{\prime}\right)-\mathbf{q}\left(\phi\left(s^{\prime}\right)\right)\right|$,

since $\phi$ is onto. Thus it follows from Equations (2)-(3) that

$$
d_{H}(\mathbf{c}, \mathbf{q}) \leq \max _{0 \leq s \leq 1}|\mathbf{c}(s)-\mathbf{q}(\phi(s))| .
$$

Using the inequality (4) we present a new bound of the Hausdorff distance $d_{H}(\mathbf{c}, \mathbf{r})$ between the conic $\mathbf{r}(t)$ with control points $\mathbf{p}_{\mathbf{0}}, \mathbf{p}_{1}, \mathbf{p}_{2}$ and the plane curve $\mathbf{c}(s)$ contained in the triangle $\triangle \mathbf{p}_{0} \mathbf{p}_{1} \mathbf{p}_{2}$, in the following proposition.

Proposition 2.2. Suppose that $\mathbf{c}:[0,1] \rightarrow \mathbf{R}^{2}$ is any continuous curve which lies entirely inside the (closed) triangle $\triangle \mathbf{p}_{0} \mathbf{p}_{1} \mathbf{p}_{2}$ and such that $\mathbf{c}(0)=\mathbf{p}_{0}$ and $\mathbf{c}(1)=\mathbf{p}_{2}$. Then

$$
d_{H}(\mathbf{c}, \mathbf{r}) \leq \frac{1}{2(1+\mu)} \max _{s \in[0,1]}\left|f_{1}(\mathbf{c}(s)) \| \mathbf{p}_{0}+\mathbf{p}_{2}-2 \mathbf{p}_{1}\right|=: \varepsilon(\mathbf{c}, \mathbf{r}) .
$$


Proof. For each point $c(s), s \in(0,1)$, there exist two intersection points of the boundary of $\Delta \mathbf{p}_{0} \mathbf{p}_{1} \mathbf{p}_{2}$ and the line passing through the point $\mathbf{c}(s)$ along the direction $\mathbf{p}_{0}+\mathbf{p}_{2}-2 \mathbf{p}_{1}$, as shown in Figure 1 . We denote the intersection point on the line segment $\overline{\mathbf{p}_{0} \mathbf{p}_{2}}$ by $\mathbf{p}_{3}$, and the other by $\mathbf{p}_{4}$. Thus we have

$$
\mathbf{p}_{3}=(1-u) \mathbf{p}_{0}+u \frac{\mathbf{p}_{0}+\mathbf{p}_{2}}{2} \quad \text { and } \quad \mathbf{p}_{4}=(1-u) \mathbf{p}_{0}+u \mathbf{p}_{1},
$$

or

$$
\mathbf{p}_{3}=(1-u) \mathbf{p}_{2}+u \frac{\mathbf{p}_{0}+\mathbf{p}_{2}}{2} \text { and } \mathbf{p}_{4}=(1-u) \mathbf{p}_{2}+u \mathbf{p}_{1},
$$

for some $u \in[0,1]$. Let $h:[0,1] \rightarrow \mathbf{R}$ be the restriction of $f_{1}$ to the line segment $\overline{\mathbf{p}_{3} \mathbf{p}_{4}}$

$$
h(\xi)=f_{1}\left((1-\xi) \mathbf{p}_{3}+\xi \mathbf{p}_{4}\right),
$$

$\xi \in[0,1]$. By Equations (6)-(7), we have

$$
h(\xi)=f_{1}\left(\frac{2-u-u \xi}{2} \mathbf{p}_{2 i}+u \xi \mathbf{p}_{1}+\frac{(1-\xi) u}{2} \mathbf{p}_{2-2 i}\right),
$$

for $i=0$ or 1. It follows from Equations (1) and (8) that

$$
h(\xi)=u \xi-\mu \sqrt{(2-u-u \xi)(1-\xi) u}
$$

and its derivative

$$
h^{\prime}(\xi)=u+\frac{\mu(1-u \xi) u}{\sqrt{(2-u-u \xi)(1-\xi) u}}
$$

for all $\xi \in[0,1]$. Since

$$
\sqrt{(2-u-u \xi)(1-\xi) u} \leq \frac{(2-u-u \xi)+(1-\xi) u}{2}=1-u \xi
$$

for all $u, \xi \in[0,1]$, we have

$$
h^{\prime}(\xi) \geq u(1+\mu) .
$$

Since $\mathbf{r}$ and $\mathbf{c}$ are continuous and $\mathbf{r}(i)=\mathbf{c}(i)=\mathbf{p}_{2 i}, i=0,1$, for each $s \in[0,1\}$ there exists $t \in[0,1]$ such that $\mathbf{r}(t)$ and $\mathbf{c}(s)$ lie on the same line segment $\overline{\mathbf{p}_{3} \mathbf{p}_{4}}$ and

$$
\mathbf{r}(t)=\left(1-\xi_{0}\right) \mathbf{p}_{3}+\xi_{0} \mathbf{p}_{4} \quad \text { and } \quad \mathbf{c}(s)=\left(1-\xi_{1}\right) \mathbf{p}_{3}+\xi_{1} \mathbf{p}_{4}
$$

for some $\xi_{0}, \xi_{1} \in(0,1)$. By the mean value theorem and Equation (9),

$$
\left|h\left(\xi_{1}\right)-h\left(\xi_{0}\right)\right| \geq \min _{\xi}\left|h^{\prime}(\xi)\right|\left|\xi_{1}-\xi_{0}\right| \geq u(1+\mu)\left|\xi_{1}-\xi_{0}\right| .
$$

Thus we have

$$
\mid \mathbf{c}(s)-\mathbf{r}(t)\}=\left|\xi_{1}-\xi_{0}\right|\left\langle\mathbf{p}_{3}-\mathbf{p}_{4}\left|\leq \frac{\left|h\left(\xi_{1}\right)\right|}{u(1+\mu)}\right| \mathbf{p}_{3}-\mathbf{p}_{4}\right|
$$


since $h\left(\xi_{0}\right)=0$ or equivalently $f_{1}(\mathbf{r}(t))=0$. By $\left|\mathbf{p}_{3}-\mathbf{p}_{4}\right|=u \mid \mathbf{p}_{0}+\mathbf{p}_{2}-$ $2 \mathbf{p}_{1} \mid / 2$ and $h\left(\xi_{1}\right)=f_{1}(\mathbf{c}(s))$, we get

$$
|\mathbf{c}(s)-\mathbf{r}(t)| \leq \frac{1}{2+2 \mu}\left|f_{1}(\mathbf{c}(s))\right|\left|\mathbf{p}_{0}+\mathbf{p}_{2}-2 \mathbf{p}_{1}\right| .
$$

Hence the required estimate (5) follows from Equations (4) and (10) by taking the maximum over $s \in[0,1]$.

Floater $[1,13]$ suggested the error bound by

$$
d_{H}(\mathbf{c}, \mathbf{r}) \leq \frac{1}{4} \max \left\{\frac{1}{\mu^{2}}, 1\right\} \max _{s \in[0,1]}\left|f_{0}(\mathbf{c}(s))\right|\left|\mathbf{p}_{0}+\mathbf{p}_{2}-2 \mathbf{p}_{1}\right|=: \psi(\mathbf{c}, \mathbf{r}) .
$$

\section{Cubic interpolations for conic sections}

In this section we apply Proposition 2.2 to cubic interpolations for conic sections whose error functions have the maximum at the midpoint of parameter. Let $\mathbf{b}(t)$ be the cubic $G^{1}$ end-points interpolation represented by

where

$$
\mathbf{b}(t):=\sum_{i=0}^{3} B_{i}^{3}(t) \mathbf{b}_{i}, \quad t \in[0,1],
$$

(12) $\mathbf{b}_{0}=\mathbf{p}_{0}, \quad \mathbf{b}_{3}=\mathbf{p}_{2}, \quad \mathbf{b}_{1}=(1-\lambda) \mathbf{p}_{0}+\lambda \mathbf{p}_{1}, \quad \mathbf{b}_{2}=(1-\lambda) \mathbf{p}_{2}+\lambda \mathbf{p}_{\mathbf{1}}$.

The parameter $\lambda(0<\lambda<1)$ determines the cubic curve

$$
\mathbf{b}(t)=\left(B_{0}^{3}+(1-\lambda) B_{1}^{3}\right) \mathbf{p}_{0}+\lambda\left(B_{1}^{3}+B_{2}^{3}\right) \mathbf{p}_{1}+\left((1-\lambda) B_{2}^{3}+B_{3}^{3}\right) \mathbf{p}_{2},
$$

and its error function

$$
\begin{aligned}
& f_{0}(\mathbf{b}(t))= \\
& t^{2}(1-t)^{2}\left[9 \lambda^{2}-4 \mu^{2}\{(1-t)+3(1-\lambda) t\}\{t+3(1-\lambda)(1-t)\}\right] \\
& \text { (13) } f_{1}(\mathbf{b}(t))= \\
& t(1-t)[3 \lambda-2 \mu \sqrt{\{(1-t)+3(1-\lambda) t\}\{t+3(1-\lambda)(1-t)\}}]
\end{aligned}
$$

In the following proposition, we show that the error bound $\varepsilon(\mathbf{b}, \mathbf{r})$ is sharper than $\psi(\mathbf{b}, \mathbf{r})$ when the error functions $f_{0}(\mathbf{b}(t))$ and $f_{1}(\mathbf{b}(t))$ have the maximum at the mid-point of parameter.

Proposition 3.1. If $f_{0}(\mathbf{b}(t))$ and $f_{1}(\mathbf{b}(t))$ have the maximum at $t=1 / 2$, then

$$
\varepsilon(\mathbf{b}, \mathbf{r}) \leq \psi(\mathbf{b}, \mathbf{r}) .
$$


Proof. By Equation (13), we have

$$
\begin{aligned}
f_{0}(\mathbf{b}(1 / 2)) & =\frac{1}{16}\left\{9 \lambda^{2}-\mu^{2}(4-3 \lambda)^{2}\right\} \\
f_{1}(\mathbf{b}(1 / 2)) & =\frac{1}{4}\{3 \lambda-\mu(4-3 \lambda)\} .
\end{aligned}
$$

Thus

$$
\begin{aligned}
\frac{\psi(\mathbf{b}, \mathbf{r})}{\varepsilon(\mathbf{b}, \mathbf{r})} & =\frac{1+\mu}{8}\{3 \lambda+\mu(4-3 \lambda)\} \max \left\{1 / \mu^{2}, 1\right\} \\
& = \begin{cases}\frac{1+\mu}{8}\{3 \lambda+\mu(4-3 \lambda)\}>\frac{1+\mu}{2}>1 & \text { if } \mu>1 \\
\frac{1+1 / \mu}{8}\{3 \lambda(1 / \mu-1)+4\}>1 & \text { if } \mu<1\end{cases}
\end{aligned}
$$

and the assertion is obtained.

The functions $f_{0}$ and $f_{1}$ have the maximum at $t=1 / 2$ for some $\lambda(\mu)$ such as in the following Propositions 3.2-3.4. Note that all cubic Bézier curves with $G^{l}$ end-point interpolation of the conic section have the control points in forms of

$$
\mathbf{b}_{0}=\mathbf{p}_{0}, \quad \mathbf{b}_{3}=\mathbf{p}_{2}, \quad \mathbf{b}_{1}=\left(1-\lambda_{1}\right) \mathbf{p}_{0}+\lambda_{1} \mathbf{p}_{1}, \quad \mathbf{b}_{2}=\left(1-\lambda_{2}\right) \mathbf{p}_{2}+\lambda_{2} \mathbf{p}_{1}
$$

for $\lambda_{1}, \lambda_{2} \in(0,1)$. But the barycentric coordinates of $\mathbf{r}(t)$ and $\mathbf{b}_{\lambda_{1}, \lambda_{2}}(t)$ for $\lambda_{1}=\lambda_{2}$, are symmetric with respect to the line passing two points $\mathbf{p}_{1}$ and $\left(\mathbf{p}_{0}+\mathbf{p}_{2}\right) / 2$, and both error bounds $\varepsilon(\mathbf{b}, \mathbf{r})$ and $\psi(\mathbf{b}, \mathbf{r})$ as in Equations (5) and (11) depend on the barycentric coordinates and the length $\left|\mathbf{p}_{0}+\mathbf{p}_{2}-2 \mathbf{p}_{1}\right|$, not on the difference of length of two legs $\left|\mathbf{p}_{0}-\mathbf{p}_{1}\right|$ and $\left|\mathbf{p}_{1}-\mathbf{p}_{2}\right|$. Hence we deal only the case of $\lambda_{1}=\lambda_{2}$, i.e., the inner control points of the cubic $G^{1}$ end-points interpolation $\mathbf{b}(t)$ in this paper are the same as in Equation (12).

Thus the error function $f_{1}(\mathbf{b}(t))$ has the symmetry with respect to $t=1 / 2$. For the simplicity, putting $\theta:=t(1-t)$ for $t \in[0,1 / 2]$ and $F(\theta):=f_{1}(\mathbf{b}(t))$ for $\theta \in[0,1 / 4\}$, we have

$$
F(\theta)=\theta\left[3 \lambda-2 \mu \sqrt{(3 \lambda-2)^{2} \theta+3-3 \lambda}\right] .
$$

In the following propositions, we present the maximum error bounds for the variant cubic interpolations having the parameter $\lambda$.

Proposition 3.2. Set $\lambda(\mu)=2 \mu /\left(\mu+\sqrt{\mu^{2}+3}\right)$. Then $\mathbf{b}$ is a $G^{2}$ Hermite interpolation of $\mathbf{r}$, and the Hausdorff distance between the two 


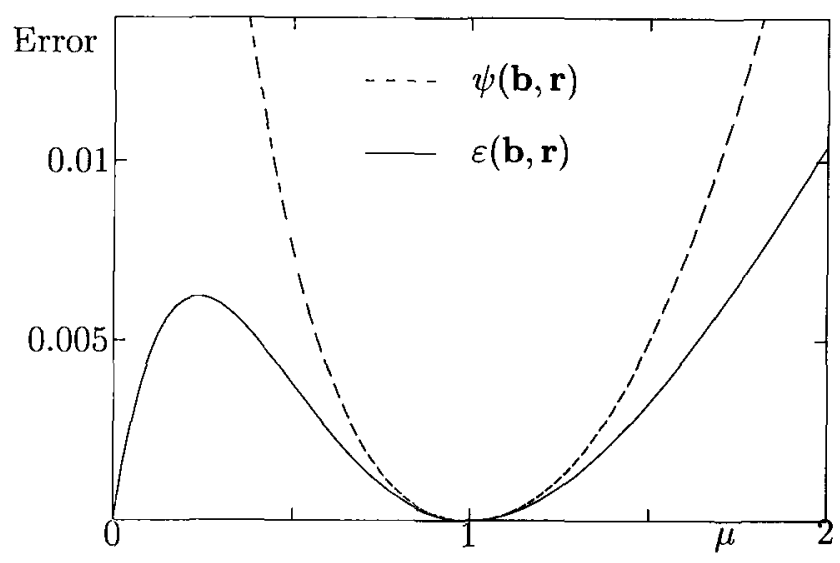

Figure 2. Two error functions $\varepsilon(\mathbf{b}, \mathbf{r})$ and $\psi(\mathbf{b}, \mathbf{r})$ for conic approximation with $\lambda(\mu)=2 \mu /\left(\mu+\sqrt{\mu^{2}+3}\right)$, where $\left|\mathbf{p}_{0}+\mathbf{p}_{2}-2 \mathbf{p}_{1}\right|=1$.

curves $\mathbf{r}$ and $\mathbf{b}$ is bounded by

$$
d_{H}(\mathbf{b}, \mathbf{r}) \leq \varepsilon(\mathbf{b}, \mathbf{r})=\frac{\mu\left(\mu+1-\sqrt{3+\mu^{2}}\right)^{2}}{8(1+\mu)}\left|\mathbf{p}_{0}+\mathbf{p}_{2}-2 \mathbf{p}_{1}\right| .
$$

Proof. It is well-known $[1,18]$ that $\mathbf{b}(t)$ is a $G^{2}$ end-points interpolation of $\mathbf{r}(t)$. By Equation (14) we have

$$
F(\theta)=\frac{-2 \mu \theta}{\mu+\sqrt{\mu^{2}+3}}\left[\sqrt{9+4 \theta\left(5 \mu^{2}+3-4 \mu \sqrt{\mu^{2}+3}\right)}-3\right] .
$$

Since

$$
4 \mu \sqrt{\mu^{2}+3} \leq \frac{8 \mu^{2}+2\left(\mu^{2}+3\right)}{2}=5 \mu^{2}+3,
$$

the equation in the bracket of Equation (15) is increasing as a function of $\theta$ and is positive. Thus $|F(\theta)|$ is increasing and has the maximum at $\theta=1 / 4$, i.e.,

$$
\left|f_{1}(\mathbf{b}(t))\right|=|F(\theta)| \leq|F(1 / 4)|=\frac{\mu\left(\mu+1-\sqrt{3+\mu^{2}}\right)^{2}}{4}
$$

for all $t \in[0,1 / 2]$. Thus by Proposition 2.2, we have the assertions. 
Remark. Put $\lambda(\mu)=2 \mu /\left(\mu+\sqrt{\mu^{2}+3}\right)$. Then $f_{0}(\mathbf{b}(t))$ is a polynomial of degree six and its absolute maximum is obtained at $t=1 / 2$, and

$d_{H}(\mathbf{b}, \mathbf{r}) \leq \psi(\mathbf{b}, \mathbf{r})=\frac{\left(\mu^{2}+1-\mu \sqrt{\mu^{2}+3}\right)^{2}}{16} \max \left\{1, \mu^{2}\right\}\left|\mathbf{p}_{0}+\mathbf{p}_{2}-2 \mathbf{p}_{1}\right|$.

This error bound $\psi(\mathbf{b}, \mathbf{r})$ is obtainable by the error analysis in [1]. As shown in Figure 2, $\varepsilon(\mathbf{b}, \mathbf{r})$ is a sharper error bound than $\psi(\mathbf{b}, \mathbf{r})$.

We adopt the method of subdivision of conic section proposed by Floater $[1,12,13]$. At any step, every conic segment is subdivided at its shoulder point into two segments, so that at each step $r$, the conic $\mathbf{r}$ consists of the $2^{r}$ segments. Under the subdivision scheme, the cubic approximate spline with $\lambda(\mu)=2\left(6 \mu^{2}+1-\sqrt{3 \mu^{2}+1}\right) /\left(12 \mu^{2}+3\right)$ of the conic section $\mathbf{r}$ is $G^{4}$. (Refer to [1].)

Proposition 3.3. Set $\lambda(\mu)=2\left(6 \mu^{2}+1-\sqrt{3 \mu^{2}+1}\right) /\left(12 \mu^{2}+3\right)$. Then

$$
d_{H}(\mathbf{r}, \mathbf{p}) \leq \frac{2 \mu^{3}-6 \mu^{2}+\mu-1+(\mu+1) \sqrt{3 \mu^{2}+1}}{4(\mu+1)\left(4 \mu^{2}+1\right)}\left|\mathbf{p}_{0}+\mathbf{p}_{2}-2 \mathbf{p}_{1}\right| .
$$

Proof. Putting $F(\theta)$ as in Equation (14) we have

$$
f_{1}(\mathbf{b}(t))=F(\theta)=\frac{2 \theta}{4 \mu^{2}+1}\left[6 \mu^{2}+1-\sqrt{3 \mu^{2}+1}-\mu \sqrt{F_{1}(\theta)}\right]
$$

for $\theta \in[0,1 / 4]$, where

$$
F_{1}(\theta)=4 \theta\left(2 \mu^{2}-\sqrt{3 \mu^{2}+1}\right)^{2}+\left(4 \mu^{2}+1\right)\left(1+2 \sqrt{3 \mu^{2}+1}\right) .
$$

Since $F_{1}(\theta)$ is a linear increasing function and

$$
\left[6 \mu^{2}+1-\sqrt{3 \mu^{2}+1}-\mu \sqrt{F_{1}(0)}\right] \leq 0
$$

for all $\mu>0, F(\theta)$ is negative for all $\theta \in[0,1 / 4]$, and also has absolute maximum at $\theta=1 / 4$, i.e.,

$$
\left|f_{1}(\mathbf{b}(t))\right| \leq|F(1 / 4)|=\frac{2 \mu^{3}-6 \mu^{2}+\mu-1+(\mu+1) \sqrt{3 \mu^{2}+1}}{2\left(1+4 \mu^{2}\right)}
$$

for all $t \in[0,1]$. Thus, by Proposition 2.2, we have the assertion.

REMARK. Put $\lambda(\mu)=2\left(6 \mu^{2}+1-\sqrt{3 \mu^{2}+1}\right) /\left(12 \mu^{2}+3\right)$. Then $f_{0}(\mathbf{b}(t))$ is a polynomial of degree six and its absolute maximum obtained 


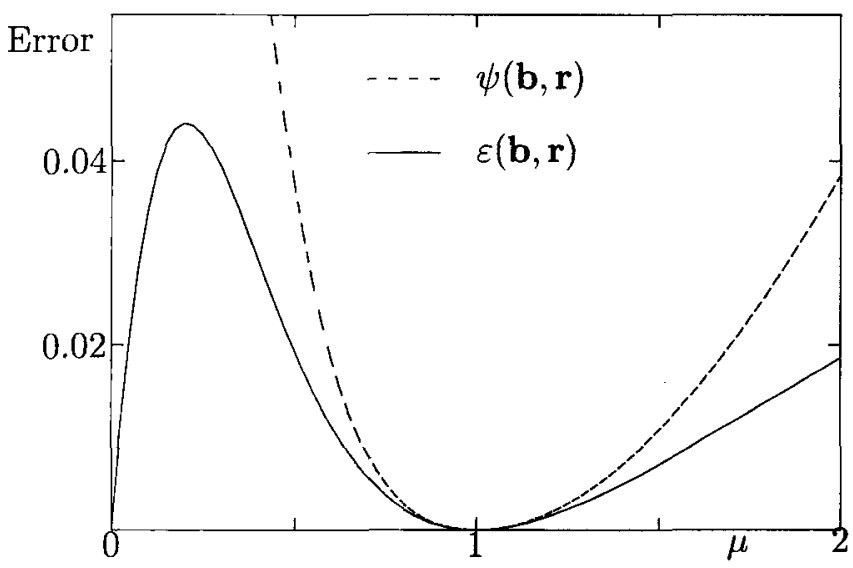

Figure 3. Two error functions $\varepsilon(\mathbf{b}, \mathbf{r})$ and $\psi(\mathbf{b}, \mathbf{r})$ for conic approximation with $\lambda(\mu)=2\left(6 \mu^{2}+1-\right.$ $\left.\sqrt{3 \mu^{2}+1}\right) /\left(12 \mu^{2}+3\right)$, where $\left|\mathbf{p}_{0}+\mathbf{p}_{2}-2 \mathbf{p}_{1}\right|=1$.

at $t=1 / 2$, and

$$
\begin{aligned}
& d_{H}(\mathbf{b}, \mathbf{r}) \leq \psi(\mathbf{b}, \mathbf{r}) \\
= & \frac{4 \mu^{6}-29 \mu^{4}-13 \mu^{2}-2+2\left(2 \mu^{4}+7 \mu^{2}+1\right) \sqrt{3 \mu^{2}+1}}{4\left(4 \mu^{2}+1\right)^{2}} \\
& \times \frac{\max \left\{1, \mu^{2}\right\}}{4 \mu^{2}}\left|\mathbf{p}_{0}+\mathbf{p}_{2}-2 \mathbf{p}_{1}\right| .
\end{aligned}
$$

This error bound $\psi(\mathbf{b}, \mathbf{r})$ is obtainable by the error analysis in [1]. As shown in Figure $3, \varepsilon(\mathbf{b}, \mathbf{r})$ is a sharper error bound than $\psi(\mathbf{b}, \mathbf{r})$.

Note [1] that the cubic approximate spline with $\lambda(\mu)=2 \mu^{2} /\left(2 \mu^{2}+1\right)$ of the conic section $\mathbf{r}$ is $C^{2}$.

Proposition 3.4. Set $\lambda(\mu)=2 \mu^{2} /\left(2 \mu^{2}+1\right)$. Then the Hausdorff distance $d_{H}(\mathbf{r}, \mathbf{p})$ is bounded by

$$
\varepsilon(\mathbf{b}, \mathbf{r})= \begin{cases}\frac{\left|A_{1}(\mu)\right|}{2(1+\mu)}\left|\mathbf{p}_{0}+\mathbf{p}_{2}-2 \mathbf{p}_{1}\right| & \text { if } \mu \leq \mu_{0} \\ \frac{\max \left\{\left|A_{1}(\mu)\right|,\left|A_{2}(\mu)\right|\right\}}{2(1+\mu)}\left|\mathbf{p}_{0}+\mathbf{p}_{2}-2 \mathbf{p}_{1}\right| & \text { if } \mu>\mu_{0}\end{cases}
$$


where

$$
\mu_{0}=\frac{1}{3}\left(1+(37+9 \sqrt{17})^{1 / 3}-2(37+9 \sqrt{17})^{-1 / 3}\right) \approx 1.575
$$

and

$$
\begin{aligned}
& A_{1}(\mu)=\frac{-\mu(\mu-1)(\mu-2)}{2\left(2 \mu^{2}+1\right)} \\
& A_{2}(\mu)=\frac{\mu}{\mu\left(5 \mu^{2}+3\right)+\left(3 \mu^{2}+1\right)^{3 / 2}} .
\end{aligned}
$$

Proof. By Equation (14) we have

$$
F(\theta)=\frac{2 \mu}{2 \mu^{2}+1} F_{2}(\theta)
$$

where $F_{2}(\theta)=\theta\left(3 \mu-\sqrt{F_{3}(\theta)}\right)$ and

$$
F_{3}(\theta)=3\left(1+\mu^{2}\right)+4 \theta\left(1-\mu^{2}\right)^{2} .
$$

For $\mu<1$, since $3 \mu-\sqrt{F_{3}(\theta)}$ is negative decreasing, $\left|F_{2}(\theta)\right|$ has its maximum at $\theta=1 / 4$.

For $\mu>1$, we have

$$
F_{2}^{\prime}(\theta)=\frac{-3}{\sqrt{F_{3}(\theta)}}\left\{2 \mu^{2}+1+2 \theta\left(1+\mu^{2}\right)^{2}-\mu \sqrt{F_{3}(\theta)}\right\}
$$

which has a unique positive real zero

$$
\theta_{0}=\frac{\mu \sqrt{1+3 \mu^{2}}-\left(1+\mu^{2}\right)}{2\left(\mu^{2}-1\right)^{2}}=\frac{2 \mu^{2}+1}{2\left(\mu^{2}-1\right)\left(\mu \sqrt{3 \mu^{2}+1}+\mu^{2}+1\right)},
$$

which is strictly decreasing from $\infty$ to zero as $\mu$ is increasing from 1 to $\infty$. Thus we have unique root $\mu=\mu_{0}$ satisfying the equation $\theta_{0}=1 / 4$. For $\mu \leq \mu_{0},\left|F_{2}(\theta)\right|$ has its maximum at $\theta=1 / 4$, and for $\mu>\mu_{0},\left|F_{2}(\theta)\right|$ has the local maximum at $\theta_{0}$.

Thus, for $\mu \leq \mu_{0},|F(\theta)|$ has the maximum $|F(1 / 4)|$, and for $\mu>\mu_{0}$, the maximum of $|F(\theta)|$ equals $\max \left\{|F(1 / 4)|,\left|F\left(\theta_{0}\right)\right|\right\}$. Since $F(1 / 4)=$ $A_{1}(\mu)$ and $F\left(\theta_{0}\right)=A_{2}(\mu)$, the assertion follows.

Remark. Put $\lambda(\mu)=2 \mu^{2} /\left(2 \mu^{2}+1\right)$. Then the sixth degree polynomial

$$
f_{0}(\mathbf{b}(t))=\frac{4 \mu^{2}\left(\mu^{2}-1\right)}{\left(2 \mu^{2}+1\right)^{2}}\left[3-4\left(\mu^{2}-1\right) t(1-t)\right] t^{2}(1-t)^{2}
$$




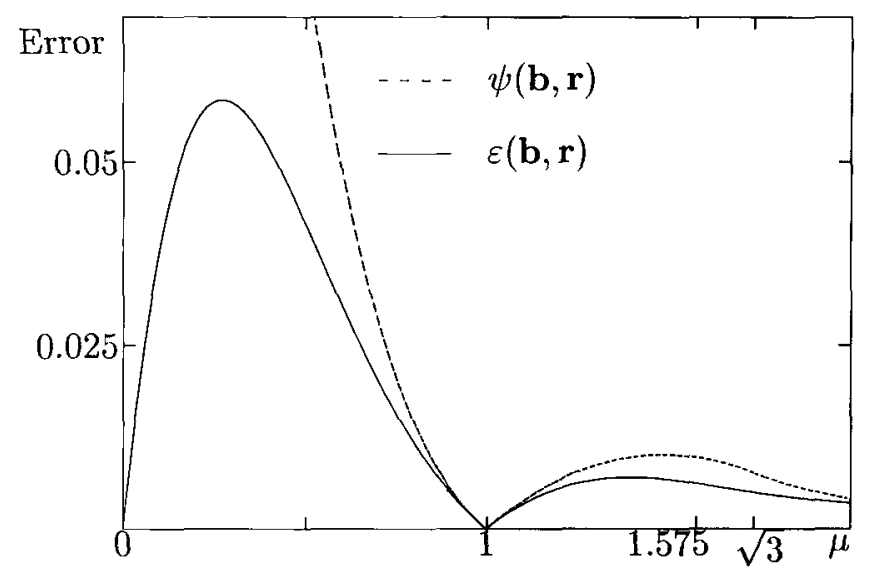

Figure 4. Two error functions $\varepsilon(\mathbf{b}, \mathbf{r})$ and $\psi(\mathbf{b}, \mathbf{r})$ for conic approximation with $\lambda(\mu)=2 \mu^{2} /\left(2 \mu^{2}+1\right)$, where $\left|\mathbf{p}_{0}+\mathbf{p}_{2}-2 \mathbf{p}_{1}\right|=1$.

is monotone in $(0,1 / 2)$ for $\mu \leq \sqrt{3}$, so that it has the unique local extremum

$$
f(\mathbf{b}(1 / 2))=-\frac{\mu^{2}\left(\mu^{2}-1\right)\left(\mu^{2}-4\right)}{4\left(2 \mu^{2}+1\right)^{2}},
$$

in $(0,1)$. For $\mu>\sqrt{3}, f_{0}(\mathbf{b}(t))$ has a unique local extremum $\mu^{2} /\left(2 \mu^{2}+\right.$ $1)^{2}\left(\mu^{2}-1\right)$ at the point satisfying $t(1-t)=1 / 2\left(\mu^{2}-1\right)$ in $(0,1 / 2)$. Thus the Hausdorff distance $d_{H}(\mathbf{r}, \mathbf{p})$ is bounded by

$$
\begin{aligned}
& \psi(\mathbf{b}, \mathbf{r}) \\
& =\left\{\begin{array}{l}
\frac{1}{4} \max \left\{\frac{1}{\mu^{2}}, 1\right\} \frac{\left|\mu^{2}\left(\mu^{2}-1\right)\left(\mu^{2}-4\right)\right|}{4\left(2 \mu^{2}+1\right)^{2}}\left|\mathbf{p}_{0}+\mathbf{p}_{2}-2 \mathbf{p}_{1}\right| \text { if } \mu \leq \sqrt{3} \\
\frac{1}{4} \frac{\mu^{2}}{\left(2 \mu^{2}+1\right)^{2}\left(\mu^{2}-1\right)}\left|\mathbf{p}_{0}+\mathbf{p}_{2}-2 \mathbf{p}_{1}\right| \text { if } \mu>\sqrt{3} .
\end{array}\right.
\end{aligned}
$$

This error bound $\psi(\mathbf{b}, \mathbf{r})$ is obtainable by the error analysis in [1]. As shown in Figure $4, \varepsilon(\mathbf{b}, \mathbf{r})$ is a sharper error bound than $\psi(\mathbf{b}, \mathbf{r})$. 


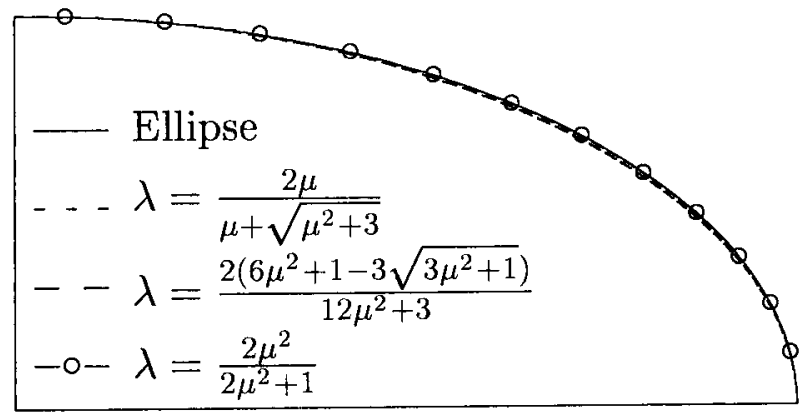

Figure 5. A quarter of the ellipse plotted by solid lines and its approximate cubic Bézier curves plotted by dash-lines or circles with $\lambda(\mu)=\frac{2 \mu}{\mu+\sqrt{\mu^{2}+3}}, \lambda(\mu)=$ $\frac{2\left(6 \mu^{2}+1-3 \sqrt{3 \mu^{2}+1}\right)}{12 \mu^{2}+3}$ and $\lambda(\mu)=\frac{2 \mu^{2}}{2 \mu^{2}+1}$, respectively.

\section{Examples}

In this section, we present the cubic interpolations of ellipse and the error functions. Let $\mathbf{r}(t)$ be the conic section with control points $(2,0)$, $(2,1)$ and $(0,1)$, in order, and weight $\mu=1 / \sqrt{2}$, which yields a quarter of ellipse in the first quadrant, as shown in Figure 5 . We also plot the $G^{1}$ cubic approximation Bézier curves $\mathbf{b}(t)$ having $\lambda(\mu)=2 \mu /\left(\mu+\sqrt{\mu^{2}+3}\right)$, $\lambda(\mu)=2\left(6 \mu^{2}+1-3 \sqrt{3 \mu^{2}+1}\right) /\left(12 \mu^{2}+3\right)$, and $\lambda(\mu)=2 \mu^{2} /\left(2 \mu^{2}+1\right)$, respectively, in Figure 5. In Figure 6, we plot the error functions $\frac{1}{2(\mu+1)} f_{1}(\mathbf{b}(t))\left|\mathbf{p}_{0}+\mathbf{p}_{2}-2 \mathbf{p}_{1}\right|$ by solid lines, and $\frac{1}{4} \max \left\{\frac{1}{\mu^{2}}, 1\right\} f_{0}(\mathbf{b}(t)) \mid \mathbf{p}_{0}+$ $\mathbf{p}_{2}-2 \mathbf{p}_{1} \mid$ by dash-lines, whose maximum norms are equal to $\varepsilon(\mathbf{b}, \mathbf{r})$ and $\psi(\mathbf{b}, \mathbf{r})$, respectively. We can see that the maximum norm $\varepsilon(\mathbf{b}, \mathbf{r})$ is less than $\psi(\mathbf{b}, \mathbf{r})$. Although the error function $\frac{1}{2(\mu+1)}\left|f_{1}(\mathbf{b}(t))\right|\left|\mathbf{p}_{0}+\mathbf{p}_{2}-2 \mathbf{p}_{1}\right|$ is not smooth at both end points as shown in Figure 6, the cubic approximation Bézier curve $\mathbf{b}(t)$ is at least $G^{1}$ end points interpolation. 


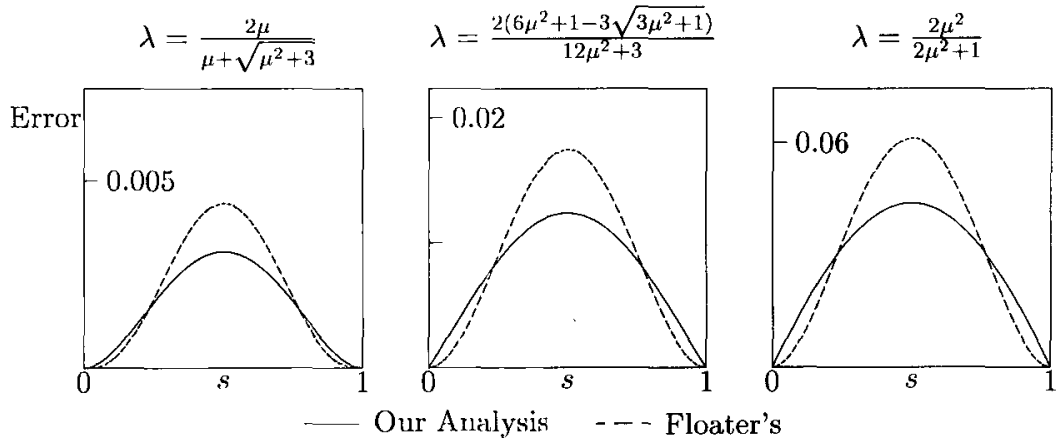

FiguRE 6. The error estimating functions using our analysis and Floater's are plotted by solid lines and dashlines, respectively.

\section{Comments}

In this paper, we presented an error analysis of spline approximation for conic section curve, and we applied it to the cubic approximations. We compared this to the error bound obtainable by the method of Floater, and we saw that our analysis gives a sharp error bounds in case the error has the maximum at the mid-point of parameter. Furthermore, this error analysis can be extended to the approximations of conic sections by quartic or quintic splines, and to the approximations of quadric surfaces by tensor product splines.

ACKNOWLEDGEMENTS. The author is very grateful to the anonymous referees for the inspiring comments and the valuable suggestions which improved our paper considerably.

\section{References}

[1] M. Floater, An analysis of cubic approximation schemes for conic sections, Advances in Computational Mathematics 5 (1996), 361-379.

[2] L. Bardis and M. Patrikalakis, Approximate conversion of rational B-spline patched, Computer Aided Geometric Design 6 (1989), 189-204.

[3] J. Hoschek, Approximate conversion of spline curves, Computer Aided Geometric Design 4 (1987), 59-66. 
[4] J. Hoschek and F. Schneider, Spline conversion for trimmed rational Bézier and B-spline surfaces, Computer Aided Design 22 (1990), 580-590.

[5] M. Patrikalakis, Approximate conversion of rational splines, Computer Aided Geometric Design 6 (1989), 155-165.

[6] T. Sederberg and M. Kakimotor, Approximating rational curves using polynomial curves, In: Farin, G., ed, NURBS for Curves and Surfaces Design, SIAM, Philadelpia, PA, 149-158.

[7] Y. J. Ahn and H. O. Kim, Approximations of circular arcs by Bézier curves, J. Computational Applied Mathematics 81 (1997), no. 1, 145-163.

[8] T. Dokken, M. Dæhlen, T. Lyche, and K. Mørken, Good approximation of circles by curvature-continuous Bézier curves, Computer Aided Geometric Design 7 (1990), 33-41.

[9] L, Fang, Circular arc approximation by quintic polynomial curves, Computer Aided Geometric Design 15 (1998), 843-861.

[10] M. Goldapp, Approximation of circular arcs by cubic polynomials, Computer Aided Geometric Design 8 (1991), 227-238.

[11] K. Mørken, Best approximation of circle segments by quadratic Bézier curves, In P. J. Laurent, A. Le Méhauté, and L. L. Schumaker, editors, Curves and Surfaces, Academic Press, New York, 1990.

[12] M. Floater, High order approximation of conic sections by quadratic splines, Computer Aided Geometric Design 12 (1995), 617-637.

[13] $\ldots$, An $\left(h^{2 n}\right)$ Hermite approximation for conic sections, Computer Aided Geometric Design 14 (1997), 135-151.

[14] G. Farin, Curves and Surfaces for Computer Aided Geometric Design, Academic Press, San Diego, CA, 1993.

[15] Y. J. Ahn and H. O. Kim, Curvatures of the quadratic rational Bézier curves, Computers and Mathematics with Applications 36 (1998), no. 9, 71-83.

[16] Y. J. Ahn, H. O. Kim and K. Y. Lee, $G^{1}$ arc spline approximations of quadratic Bézier curves, Computer-Aided Design 31 (1998), 615-620.

[17] E. T. Lee, The rational Bézier representation for conics, In geometric modeling: Algorithms and new trends, pages 3-19, SIAM, Academic Press, Philadelphia, 1987.

[18] C. de Boor, K. Höllig, and M. Sabin, High accuracy geometric Hermite interpolation, Computer Aided Geometric Design 4 (1987), 169-178.

Department of Mathematics

Ajou University

Kyunggi-do 442-749, Korea

E-mail: ahn@madang.ajou.ac.kr 